\title{
Assessment of High Sn Incorporation in Ge NanoWires Synthesized via In Plane Solid-Liquid-Solid Mechanism by In-Situ TEM
}

\author{
Simona Moldovan ${ }^{1}$, Edy Azrak ${ }^{1,2}$, Wanghua Chen ${ }^{2}$, Shiwen $\mathrm{Gao}^{2}$, Sebastien Duguay ${ }^{1}$, Philippe Pareige ${ }^{1}$ \\ and Pere Roca i Cabarrocas ${ }^{2}$ \\ 1. Groupe de Physique des Matériaux, CNRS, Normandie Université, Rouen, France. \\ 2. LPICM, CNRS, Ecole polytechnique, Université Paris-Saclay, Palaiseau, France.
}

Silicon is the most common material used to build up high performance electronics. As compared with the hybrid structures combining silicon technology and III-V materials, Germanium (Ge) and $\mathrm{Si}_{1-\mathrm{x}} \mathrm{Ge}_{\mathrm{x}}$ on $\mathrm{Si}$ are prone to considerably lower the production costs. To transform Ge in a direct gap, two main "bandgap" engineering approaches have been developed: the use of tensile strain and the Sn incorporation. In this study, we focus on this second methodology. There are few major obstacles to overcome to successfully fabricate GeSn alloys with high Sn content: i) the low $<1 \%$ solubility of Sn in Ge at equilibrium, ii) the strong Sn segregation behavior [1] and iii) the poor crystalline quality due mainly to the lattices mismatch [2]. Gupta et al. [3] employed a non-local empirical pseudopotential method to obtain a Sn concentration of $6.5-11 \%$. Successful strategies for the growth of metastable GeSn thin films were developed [2], whilst the "classical" methods led to relatively low Sn concentrations [4]. The nanowire (NW) alloy configuration has been more and more explored as in this case the growth process is governed by non-equilibrium mechanisms [5] building-up strain-relaxed [6] structures. The solid-liquid-solid (SLS) mechanism leads to $\sim 12 \%$ at Sn incorporation for NWs with low crystallinity [7]. Based on the in plane SLS (IPSLS) mechanism [8], we have employed two different methodologies to grow Ge NWs by using Sn-based particles as catalyst onto Si substrate, on which a uniform amorphous Ge layer was deposited. First, an in-situ hydrogen assisted plasma approach was employed to grow the Ge NWs and perform the ex-situ TEM.Secondly the in-situ Transmission Electron Microscopy (TEM) experiments as developed under vacuum and hydrogen environement were engaged to adress the growth mechanisms involved.

The ex-situ analysis of the synthesized NWs (Figure 1) has identified their general morphology as well as their chemical composition. The first step of growth is the reduction of the Sn-based particles under hydrogen plasma into metallic Sn, which catalyzes the NW growth. However, the reduction is only partial as at the starting point of the NW we identify a reminescent particle. Further, the NWs growth onsets at $270^{\circ} \mathrm{C}$ andthe NW follows a random direction digging a trench in the amorphous Ge. Small Sn-rich nano-particles (20 nm in average) diffuse on the surface of the amorphous Ge and finally accomodate at the trench borders. In the final stage, once the catalyst is consummed, the growth stops implying that the catalysts size "dictates" the NWs length. The chemical composition of the NWs as measured for a tens of cross-sections prepared by Focussed Ion Beam (FIB) revealed a surprisingly high content of Sn (mean 21 at\%), whilst the polycrystalline nature of the NWs combined with the morphology observations points to a strained structure. Indeed, Raman spectroscopy confirmed the substitution Sn-induced bond stretching from the Ge NWs [8], confirming the high Sn content within a strained NW. From a phenomenological perspective, during the NW growth, the decrease of the catalysts size occurs by the continous dissolution of Sn atoms into the GeSn NW, the process being governed by the flux of the Ge atoms involved and the triple phase line (est-ce pus clair?) represented in Figure 2.a). Since this approach does not explain the high Sn incorporation, two hypothesis are advanced and tested by the in-situ TEM: the step-flow kinetics (figure2.b) [9] and the dimers insertion [10]. First, 
the Sn catalyst reduces partially under the impact of the beam under vacuum and fragments in small metallic Sn NPs under hydrogen environement. Afterwards, the NW structure builds up by subsequent filling of the atomic planes, but no clear evidence of the Ge-Sn dimers was observed.

\section{References:}

[1] Kurdi et al, J of Applied Physics 107 (2010), p. 013710.

[2] Kormoš et al, Surface and Interface Analysis 49 (2017), p. 297.

[3] Gupta et al, Journal of Applied Physics 113 (2013), p. 073707.

[4] Wirths et al, Nat Photon 9 (2015), p. 88.

[5] Xiang et al, Physical Review B 78 (2008), p. 193301.

[6] Karam et al, Surface and Interface Analysis 126 (2017).

[7] Kodambaka et al, Science 316 (2007), p. 729.

[8] L. Yu and P. Roca i Cabarrocas, Phys. Rev. B 81 (2010), p. 085323.

[8] Rojas-Lopes et al, J of ppl Physics 76 (1994), p. 1518.

[9] Biswas et al, NatComm 7 (2016), p. 11405.

[10] Chen et al, NatComm 5 (2014), p. 4134.
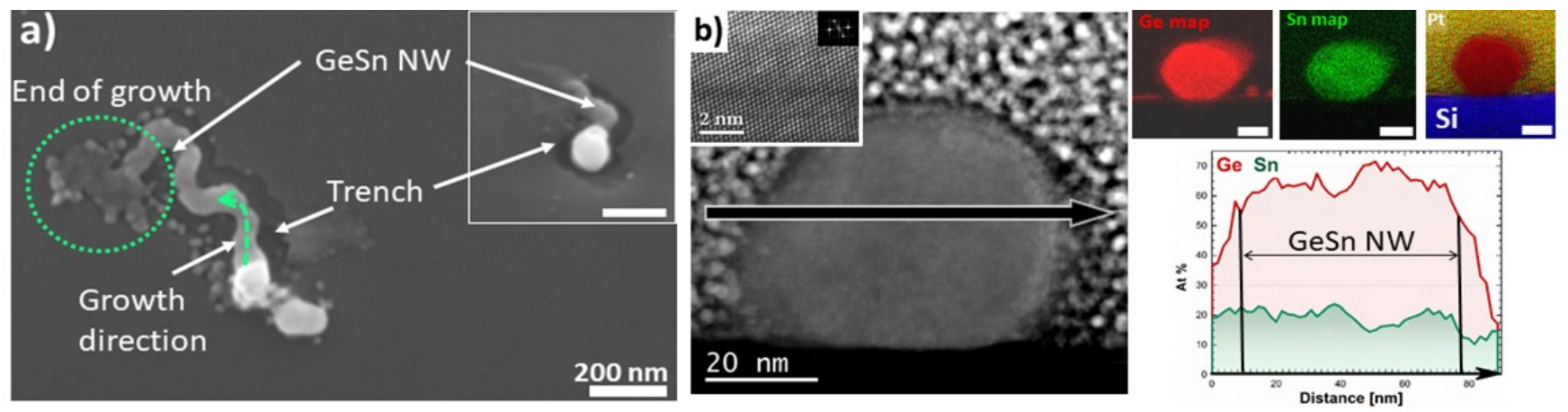

Figure 1. Morphology and microstructure of the GeSn NWs grown by hydrogen plasma assisted methodology. a) SEM plane-view micrograph of the as-grown NW; b) STEM-HAADF micrographs and STEM-EDS chemical maps within a FIB cross-section

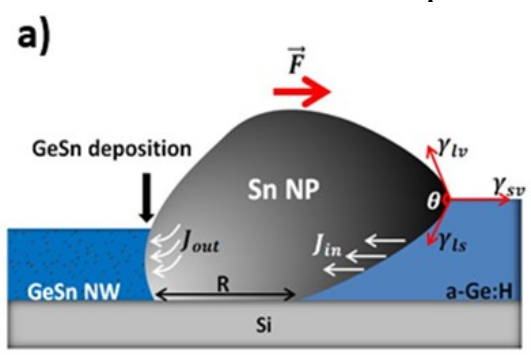

b)

Figure 2. Schematic illustrations of: a) IPSLS growth mechanism controlled by the $J_{\text {in }}$ and $J_{\text {out }}$, the absorbtion and deposition flux of Ge atoms, respectively and the surface tensions $\gamma$ of: catalystsvacuum, amorphous Ge-vacuum and catalyst-amorphous Ge; b) step-flow kinetics mechanism with the velocities of the step, atomic lines and interface 